\title{
Impact of Next-Generation Sequencing on Outcomes in Hepatocellular Carcinoma: How Precise Are We Really?
}

This article was published in the following Dove Press journal: Journal of Hepatocellular Carcinoma

\author{
Dana A Dominguez' \\ Xin Wei Wang ${ }^{1,2}$ \\ 'Laboratory of Human Carcinogenesis, \\ Center for Cancer Research, National \\ Cancer Institute, Bethesda, MD 20892, \\ USA; ${ }^{2}$ Liver Cancer Program, Center for \\ Cancer Research, National Cancer \\ Institute, Bethesda, MD 20892, USA
}

\begin{abstract}
Advances in Next-Generation Sequencing (NGS) technology have led to a rapid expansion in the application of "precision medicine," attempting to personalize therapies and improve outcomes for cancer patients. While these technologies carry great promise, evidence for translation to patient outcomes is lacking. This is especially true in hepatocellular carcinoma (HCC), where multiple factors have hampered efforts to develop effective targeted therapies. We sought to review how application of NGS in HCC has translated to patient outcomes and what barriers stand in the way of further progress.
\end{abstract}

Keywords: next generation sequencing, hepatocellular carcinoma, precision medicine, sequencing

\section{Introduction}

Hepatocellular carcinoma (HCC), the most common type of liver cancer, carries a high morbidity and mortality; in the United States alone its incidence has been increasing at an annual rate of $1-4 \%$ since $2000 .^{1}$ Implementation of surveillance and screening protocols for patients at increased risk of $\mathrm{HCC}$ (i.e. hepatitis B, C, D and cirrhosis), has allowed for the detection of earlier stage tumors which are more amenable to curative therapy and lead to improved long-term survival. However, less than half of all new HCC tumors are detected through these protocols so patients still often present at advanced stages. ${ }^{2}$ This disparity highlights the need for improvement in early detection, but also points to the need for ongoing development of effective treatment strategies in advanced disease.

Development of effective therapies for HCC has proven exceptionally difficult. Since the approval of the tyrosine kinase inhibitor (TKI) sorafenib, after demonstration of a three-month overall survival benefit in 2008, few additional therapies have been shown to improve on this benchmark. ${ }^{3} \mathrm{~A}$ handful other TKIs have been approved including lenvatinib and regorafenib, but have only been able to show noninferiority to sorafenib as first-line therapy or benefit after progression on sorafenib, respectively. ${ }^{4,5}$ The additional promise of immunotherapy through targeting of CTLA-4 and PD-1/PD-L1 have yet to demonstrate significant benefit. The CheckMate-040 trial, the only trial with results comparing immunotherapy with nivolumab, a PD-1 inhibitor, vs the standard of care sorafenib as first-line therapy, had a response rate of only $20 \%$ and failed to demonstrate significant overall survival benefit. ${ }^{6,7}$ Difficulty in development of effective therapies is likely due in

\footnotetext{
Tel +I 2407606858

Fax + I $24054 \mid 4444$

Email xw3u@nih.gov
}

Correspondence: Xin Wei Wang 
large part to the significant heterogeneity that exists in HCC. Multiple etiologies including hepatitis B, C and D, and cirrhosis contribute to different molecular subtypes of HCC which can respond differently to subsequent therapy. ${ }^{8}$ In addition, HCC has been found to have particularly robust intertumor and intratumor heterogeneity including driver mutations, highlighting the need for identification of specific patient populations that will respond to individual therapies. ${ }^{9}$

The introduction of Next-Generation Sequencing (NGS) in 2005, with the most common clinical applications being through use of targeted sequencing such as FoundationOne (Foundation Medicine, Cambridge, MA) and Oncomine panels (Thermo Fisher, Waltham, MA), as well as whole-exome sequencing (WES). These applications have significantly increased our ability to parse a tumor's individual characteristics as well as the potential for development of new targeted therapies ushering in the era of "precision medicine". ${ }^{10}$ While it has demonstrated success in certain cancers by matching patients to treatment based on individual tumor characteristics, improving response rates as well as survival in clinical trials, this promise has not yet borne out in HCC. ${ }^{11}$ Importantly, application of NGS, is not specific to probing a certain type of alteration, such as DNA mutations, but rather as a tool with which to probe the substrate of choice from the transcriptome to the epigenome. This fact highlights its powerful ability to probe a multitude of alterations in pathways of carcinogenesis. Most widely used targeted clinical NGS panels rely on the use of formalin-fixed paraffinembedded tumor tissue, however, there is significant research focus on use of other tissue types, the most provocative being "liquid biopsy" using cell free DNA (cfDNA) or circulating tumor cells (CTC). With the advancement and complexity of existing and novel sequencing technologies, it is critical to maintain definitions of "actionable targets," both generally and arguably, more importantly, within the context of each application. In a 2016 review on defining actionable mutations for oncologic therapies, Carr et al defined an actionable mutation as, "a DNA change that, if detected in a patient's tumor, would be expected (or predicted) to affect a patient's response to treatment."12 As alluded to by the authors, this broad definition necessarily varies on a study by study basis and serves as an inherent determinant of detection power within the study. This article serves to highlight the successes and, more importantly, the barriers to improving outcomes of patients with HCC using NGS technologies.

\section{What Have We Gained from NGS in HCC?}

While there have not been significant advances in terms of patient outcomes through integration of NGS into HCC management, it has provided a genetic landscape from which to build. Multiple studies have now identified driver mutations common among HCC tumors from patients with different ethnicities and etiologies. ${ }^{13-18}$ These studies identified the most prevalent driver mutations including TERT, CTNNB1, TP53, $A X I N 1, A R I D 1 A$, and ARID1B, which unfortunately are commonly seen as "non-druggable". However, these studies may establish a potential contribution of underlying tumor etiology to the unique mutational landscape of the respective tumor. $^{15,19}$

Recent work by Harding et al has managed to take NGS a step closer to affecting clinical care in HCC. Using MSKIMPACT, an FDA approved panel of 468 genes, they were able to prospectively identify mutations that portended worse outcomes both with sorafenib and immune checkpoint inhibitors. Patients with oncogenic PI3K-mTOR alterations had significantly worse outcomes than those without when treated with sorafenib. Similarly, patients with an activating mutation of $\mathrm{WNT} / \beta$-catenin were resistant to checkpoint inhibitor therapy. ${ }^{20}$ This work is particularly promising because although only around $3 \%$ of cancer drugs tested in clinical trials eventually go on to obtain FDA approval, ${ }^{21}$ the use of a biomarker for patient selection may double the chances of success. ${ }^{22}$ One could suspect that the next incremental success in the application of NGS in HCC may come from a basket trial such as CUSTOM or NCI-MATCH in which patients with various tumors are screened with NGS and those patients with specific tumor mutations are subsequently enrolled and treated with a target drug on a clinical trial. $^{23}$ Moreover, trial enrichment, by selection of patients for a trial based on their molecular profile, which has yielded advancements in other tumor types such including EGF-R or ALK positive lung cancer or wild type RAS in colorectal cancer, has potential or benefit, however, is likely to be constrained by HCC's significant molecular heterogeneity. ${ }^{24}$

\section{Barriers in Translating Data to Patient Outcomes}

Despite limited translation of NGS to direct patient benefit in $\mathrm{HCC}$, we now have a much better understanding of the 
complex framework of HCC tumors created by the unique mutational heterogeneity which has plagued efforts in targeted therapy thus far. We feel that the most important knowledge to date brought about through application of NGS in HCC has been in illuminating multiple ways by which HCC may overcome repeated attempts at one-size-fits-all therapies including chemotherapy, sorafenib and other small molecules.

HCC, compared to many other cancer types, has a low prevalence of existing or known actionable target mutations. An examination of The Cancer Genome Atlas Program (TCGA) data in comparison with the Database of Evidence for Precision Oncology (DEPO) demonstrated that of the 354 patients in the Liver Hepatocellular Carcinoma (LIHC) cohort, only $6 \%$ of patients had at least one currently druggable mutation compared to a rate of $30 \%$ in all combined cancer types. ${ }^{25}$ Further complicating the matter is the significant intertumor and intratumor heterogeneity that exists compared with other cancers (Figure 1). ${ }^{8,17,26-30}$ Intertumor heterogeneity or the harboring of unique $\mathrm{HCC}$ clones within synchronous tumors has been shown to be present anywhere from $22-60 \%$ of multicentric tumors. ${ }^{31}$ In addition, the advent of sequencing on the single-cell level has illustrated the degree of intratumor heterogeneity on a transcriptome level that exists extremely early on in tumor development. ${ }^{29,30}$ Finally, there exists a temporal heterogeneity by which the tumor genome will be altered as it progresses and undergoes various therapies. ${ }^{32}$ Utilization of higher resolution technologies including single cell will be vital in addressing the issue of heterogeneity by ensuring that targeted therapies address a broad population of cancer cells through their effector targets.

In addition to tumor-specific factors, there exist logistic barriers further hampering efforts to gain insight into this

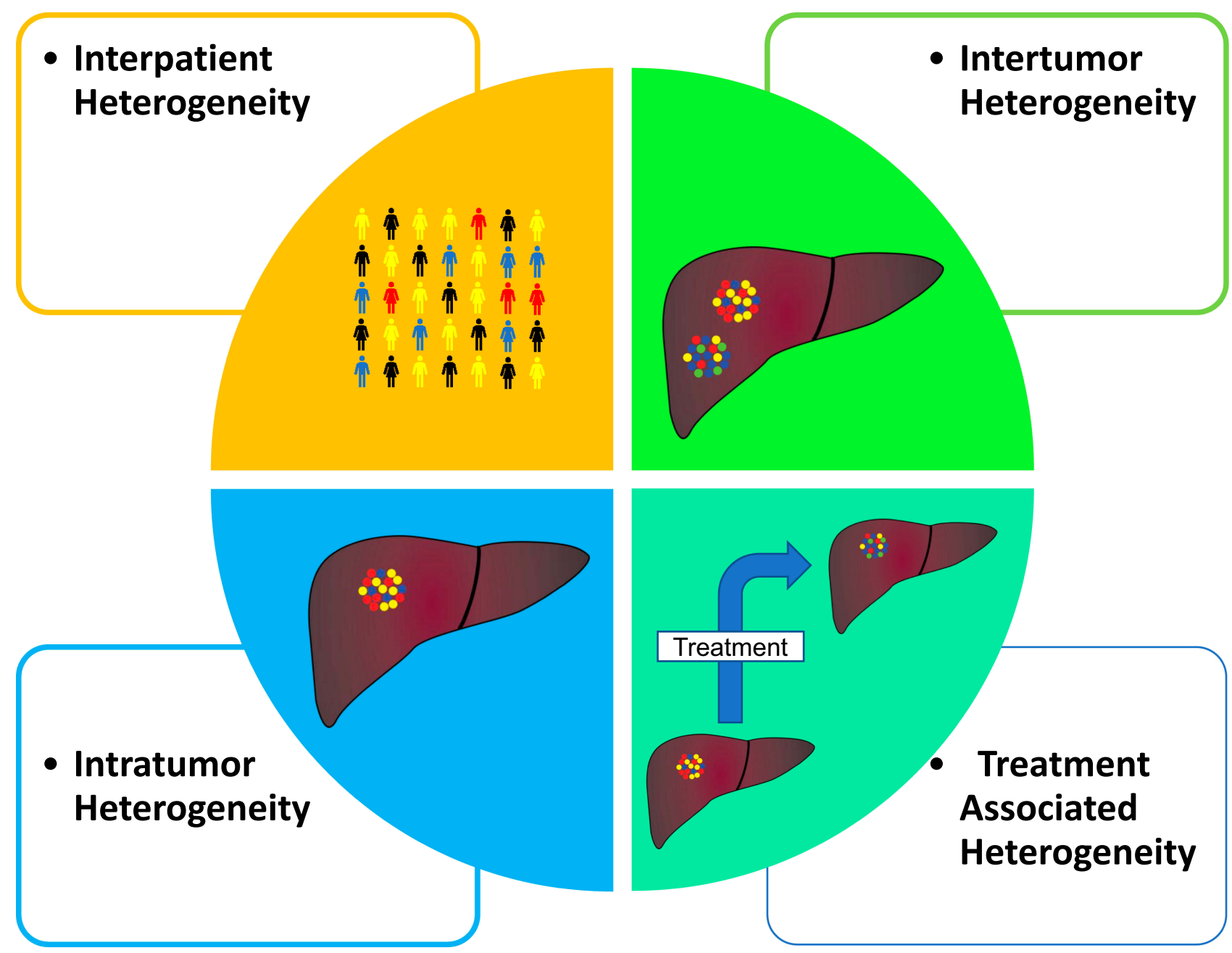

Figure I Four main types of tumor heterogeneity. Interpatient heterogeneity (top-left) describing differences between patients with similar tumor histology. Intertumor heterogeneity (top-right) describes differences between tumor foci in the same patient. Intratumor heterogeneity (bottom-left) demonstrates differences in tumor cells within the one tumor foci and lastly treatment associated heterogeneity (bottom-right) describes temporal changes in a tumor at intervals throughout treatment. 
disease. As HCC management guidelines do not require tissue diagnosis, biopsies are not always readily available for all patients with HCC. This prevents insight into the tumor environment for research purposes, and it also makes it more difficult for patients to be screened for biomarkers, such as the aforementioned alterations in $\mathrm{WNT} / \beta$-catenin or PI3K-mTOR, prior to treatment with sorafenib or checkpoint inhibitors. Although these biomarkers were identified in a small cohort of patients, their ability to predict response to therapy illustrates the importance of tissue analysis on patients with treatment refractory malignancies and warrants investigation in larger clinical trials.

\section{Where Will Advances Come from?}

The development of single cell resolution in sequencing technology has already contributed a wealth of information during its relatively short time in practice. While the recognized targetable mutation burden in $\mathrm{HCC}$ is relatively low, the increased resolution of newer NGS technologies has allowed for the discovery of striking tumor heterogeneity and has the potential to allow for better understanding of treatment resistance and the interplay of the limited targetable driver mutations that do exist.

In order to address the paucity of routine tumor biopsies at diagnosis or during therapy, there have been strides to develop "liquid biopsy" techniques aimed at detecting CTC as well as cfDNA in the patients plasma. ${ }^{33}$ Unfortunately, in HCC, the burden of CTC in the peripheral blood is believed to be small, with existing studies detecting CTC with a widely varied ability from $22-100 \%$. These results demonstrate that using existing enrichment and detection technologies, sensitivity for CTC remains poor. ${ }^{34}$ However, a recent subset study from the SORAMIC trial ( $\mathrm{Y}^{90}+$ sorafenib vs $\mathrm{Y}^{90}$ alone), applied NGS to cfDNA to predict therapy response by detecting changes in mutational variants and frequency over time in patients with advanced HCC undergoing therapy. ${ }^{35}$ While this application was only on a small subset of patients, it demonstrates the significant potential for gaining a wealth of information from an easily obtained sample.

Lastly, given the relative scarcity of HCC tissue, as well as the similarly low prevalence of currently druggable mutations highlights the importance of large NGS cohort trials such as MSK-IMPACT and NIH-MATCH which include nested basket trials that could subsequently lead to breakthroughs and significant improvements to patient outcomes in HCC. The framework of these trials allows for a standardization of NGS detection, establishes a pipeline for patient triage into treatment trials and ensures a way for patients to not only improve their own cancer care, but also to further the fundamental understanding of the disease.

\section{Conclusion}

Although application of NGS technologies in HCC has not carried with it an immediate benefit to patient outcomes, it has rapidly and fundamentally changed our understanding of the molecular complexity of the disease. It allows us to think differently about how we apply and develop new therapies, setting aside the idea of a "silver bullet" and focusing on how we can better identify tumoral or patient characteristics that will benefit from a given therapy. Despite existing barriers to development of targeted therapies, the evolution of NGS technologies and more minimally invasive biopsy techniques will undoubtedly lead to a more personalized view of treatment planning and help to realize the dream of a true precision medicine approach to HCC management.

\section{Acknowledgment}

This work was supported by grants (Z01 BC 010877, Z01 BC 010876, Z01 BC 010313) from the intramural research program of the Center for Cancer Research, National Cancer Institute, Bethesda, MD, USA.

\section{Disclosure}

The authors report no conflicts of interest in this work.

\section{References}

1. Kulik L, El-Serag HB. Epidemiology and management of hepatocellular carcinoma. Gastroenterology. 2019;156(2):477-491, e471. doi:10.1053/j.gastro.2018.08.065

2. Zhao C, Xing F, Yeo YH, et al. Only one-third of hepatocellular carcinoma cases are diagnosed via screening or surveillance: a systematic review and meta-analysis. Eur J Gastroenterol Hepatol. 2019;32 (3):406-419.

3. Llovet JM, Ricci S, Mazzaferro V, et al. Sorafenib in advanced hepatocellular carcinoma. $N$ Engl J Med. 2008;359(4):378-390. doi:10.1056/ NEJMoa0708857

4. Kudo M, Finn RS, Qin S, et al. Lenvatinib versus sorafenib in first-line treatment of patients with unresectable hepatocellular carcinoma: a randomised Phase 3 non-inferiority trial. Lancet. 2018;391 (10126):1163-1173. doi:10.1016/S0140-6736(18)30207-1

5. Bruix J, Qin S, Merle P, et al. Regorafenib for patients with hepatocellular carcinoma who progressed on sorafenib treatment (RESORCE): a randomised, double-blind, placebo-controlled, phase 3 trial. Lancet. 2017;389(10064):56-66. doi:10.1016/S0140-6736(16)32453-9

6. Bristol-Myers Squibb Announces Results from CheckMate -459 Study Evaluating Opdivo (Nivolumab) as a First-Line Treatment for Patients with Unresectable Hepatocellular Carcinoma [Press Release]. BristolMeyers Squibb; June 24, 2019.

7. El-Khoueiry AB, Sangro B, Yau T, et al. Nivolumab in patients with advanced hepatocellular carcinoma (CheckMate 040): an open-label, non-comparative, Phase 1/2 dose escalation and expansion trial. Lancet. 2017;389(10088):2492-2502. doi:10.1016/S0140-6736(17)31046-2 
8. Chaisaingmongkol J, Budhu A, Dang H, et al. Common molecular subtypes among asian hepatocellular carcinoma and cholangiocarcinoma Cancer Cell. 2017;32(1):57-70, e53. doi:10.1016/j.ccell.2017.05.009

9. Gao Q, Wang ZC, Duan M, et al. Cell culture system for analysis of genetic heterogeneity within hepatocellular carcinomas and response to pharmacologic agents. Gastroenterology. 2017;152(1):232-242, e234. doi:10.1053/j.gastro.2016.09.008

10. Karlovich CA, Williams PM. Clinical applications of next-generation sequencing in precision oncology. Cancer J. 2019;25(4):264-271. doi:10.1097/PPO.0000000000000385

11. Tsimberidou AM, Iskander NG, Hong DS, et al. Personalized medicine in a Phase I clinical trials program: the MD Anderson cancer center initiative. Clin Cancer Res. 2012;18(22):6373-6383.

12. Carr TH, McEwen R, Dougherty B, et al. Defining actionable mutations for oncology therapeutic development. Nat Rev Cancer. 2016;16(5):319-329. doi:10.1038/nrc.2016.35

13. Totoki Y, Tatsuno K, Covington KR, et al. Trans-ancestry mutational landscape of hepatocellular carcinoma genomes. Nat Genet. 2014;46 (12):1267-1273. doi:10.1038/ng.3126

14. Guichard C, Amaddeo G, Imbeaud S, et al. Integrated analysis of somatic mutations and focal copy-number changes identifies key genes and pathways in hepatocellular carcinoma. Nat Genet 2012;44(6):694-698. doi:10.1038/ng.2256

15. Schulze K, Imbeaud S, Letouze E, et al. Exome sequencing of hepatocellular carcinomas identifies new mutational signatures and potential therapeutic targets. Nat Genet. 2015;47(5):505-511. doi:10.1038/ng.3252

16. Li M, Zhao H, Zhang X, et al. Inactivating mutations of the chromatin remodeling gene ARID2 in hepatocellular carcinoma. Nat Genet. 2011;43(9):828-829. doi:10.1038/ng.903

17. The Cancer Genome Atlas Research Network. Comprehensive and integrative genomic characterization of hepatocellular carcinoma. Cell. 2017;169(7):1327-1341. doi:10.1016/j.cell.2017.05.046

18. Huang J, Deng Q, Wang Q, et al. Exome sequencing of hepatitis B virus-associated hepatocellular carcinoma. Nat Genet. 2012;44 (10):1117-1121, e1323. doi:10.1038/ng.2391

19. Chaudhary K, Poirion OB, Lu L, Huang S, Ching T, Garmire LX. Multimodal meta-analysis of 1,494 hepatocellular carcinoma samples reveals significant impact of consensus driver genes on phenotypes. Clin Cancer Res. 2019;25(2):463-472. doi:10.1158/1078-0432.CCR-18-0088

20. Harding JJ, Nandakumar S, Armenia J, et al. Prospective genotyping of hepatocellular carcinoma: clinical implications of next-generation sequencing for matching patients to targeted and immune therapies. Clin Cancer Res. 2019;25(7):2116-2126. doi:10.1158/1078-0432. CCR-18-2293

21. Lin A, Giuliano CJ, Palladino A, et al. Off-target toxicity is a common mechanism of action of cancer drugs undergoing clinical trials. Sci Transl Med. 2019;11:509. doi:10.1126/scitranslmed. aaw8412
22. Wong $\mathrm{CH}$, Siah KW, Lo AW. Estimation of clinical trial success rates and related parameters. Biostatistics. 2019;20(2):273-286. doi:10.1093/ biostatistics/kxx069

23. Cunanan KM, Gonen M, Shen R, et al. Basket trials in oncology: a trade-off between complexity and efficiency. J Clin Oncol. 2017;35 (3):271-273. doi:10.1200/JCO.2016.69.9751

24. Bruix J, da Fonseca LG, Reig M. Insights into the success and failure of systemic therapy for hepatocellular carcinoma. Nat Rev Gastroenterol Hepatol. 2019;16(10):617-630. doi:10.1038/s41575-019-0179-x

25. Bailey MH, Tokheim C, Porta-Pardo E, et al. Comprehensive characterization of cancer driver genes and mutations. Cell. 2018;174 (4):1034-1035. doi:10.1016/j.cell.2018.07.034

26. Ye QH, Qin LX, Forgues M, et al. Predicting hepatitis B virus-positive metastatic hepatocellular carcinomas using gene expression profiling and supervised machine learning. NatMed. 2003;9(4):416-423.

27. Lee JS, Chu IS, Heo J, et al. Classification and prediction of survival in hepatocellular carcinoma by gene expression profiling. Hepatology. 2004;40(3):667-676. doi:10.1002/hep.20375

28. Boyault S, Rickman DS, de Reynies A, et al. Transcriptome classification of $\mathrm{HCC}$ is related to gene alterations and to new therapeutic targets. Hepatology. 2007;45(1):42-52. doi:10.1002/hep.v45:1

29. Zheng H, Pomyen Y, Hernandez MO, et al. Single cell analysis reveals cancer stem cell heterogeneity in hepatocellular carcinoma. Hepatology. 2018;68(1):127-140. doi:10.1002/hep.29778

30. Ma L, Hernandez MO, Zhao Y, et al. Tumor cell biodiversity drives microenvironmental reprogramming in liver cancer. Cancer Cell. 2019;36:13. doi:10.1016/j.ccell.2019.08.007

31. Lu LC, Hsu CH, Hsu C, Cheng AL. Tumor heterogeneity in hepatocellular carcinoma: facing the challenges. Liver Cancer. 2016;5 (2):128-138. doi:10.1159/000367754

32. Dagogo-Jack I, Shaw AT. Tumour heterogeneity and resistance to cancer therapies. Nat Rev Clin Oncol. 2018;15(2):81-94. doi:10.1038/ nrclinonc. 2017.166

33. Martin SP, Wang XW. The evolving landscape of precision medicine in primary liver cancer. Hepat Oncol. 2019;6(2):HEP12. doi:10.2217/ hep-2019-0004

34. Ye Q, Ling S, Zheng S, Xu X. Liquid biopsy in hepatocellular carcinoma: circulating tumor cells and circulating tumor DNA. Mol Cancer. 2019;18(1):114. doi:10.1186/s12943-019-1043-x

35. Alunni-Fabbroni M, Ronsch K, Huber T, et al. Circulating DNA as prognostic biomarker in patients with advanced hepatocellular carcinoma: a translational exploratory study from the SORAMIC trial. J Transl Med. 2019;17(1):328. doi:10.1186/s12967-019-2079-9
Journal of Hepatocellular Carcinoma

\section{Publish your work in this journal}

The Journal of Hepatocellular Carcinoma is an international, peerreviewed, open access journal that offers a platform for the dissemination and study of clinical, translational and basic research findings in this rapidly developing field. Development in areas including, but not limited to, epidemiology, vaccination, hepatitis therapy, pathology and molecular tumor classification and prognostication are all considered for publication. The manuscript management system is completely online and includes a very quick and fair peer-review system, which is all easy to use. Visit http://www.dovepress.com/ testimonials.php to read real quotes from published authors. 